\title{
A PRODUÇÃO CIENTÍFICA BRASILEIRA NA WEB OF SCIENCE (2017-2019): ENTRE O DISCURSO POLÍTICO E OS ESTUDOS MÉTRICOS DE INFORMAÇÃO
}

\author{
Leilah Santiago Bufrem \\ Universidade Federal da Paraíba - UFPB \\ santiagobufrem@gmail.com
}

Bruna S. do Nascimento

Universidade Federal do Estado do Rio de Janeiro - UNIRIO

bruna.nascimento@unirio.br

\begin{abstract}
Resumo
Apresenta as características da produção científica brasileira representada na Web of Science (WOS) como exemplo ilustrativo resultante das relações entre elementos estruturais e conjunturais na dinâmica das políticas científicas adotadas pelo governo em relação à ciência brasileira. Subsidia metodologicamente o estudo relacional do campo científico no Brasil, optando pela utilização da análise de conjuntura em prol da compreensão da diversidade dos elementos empíricos representativos da produção científica brasileira indexada na WOS, entre os anos de 2017 e 2019. Analisa como as exigências produtivistas das agências de fomento se intensificaram nas últimas décadas e se fazem presentes nas discussões em diferentes campos do saber, argumentando que as rígidas práticas empregadas no campo da Ciência justificam estudos sobre as correlações e interações em prol da compreensão e do respeito ao campo e às instituições nas quais se alicerça. Apresenta como resultados, a partir dos dados empíricos, a formação de cinco grandes clusters entre os quais se destaca a Universidade de São Paulo (USP) como nó central e polo de produção científica nacional. Reconhece que, embora a base de dados escolhida, represente a big science internacionalizada e centralizada na produção oriunda dos Estados Unidos e de países europeus, a representatividade brasileira é relevante, especialmente se consideradas as temáticas de interesse endêmico como as doenças tropicais e que são pesquisadas, predominantemente, pelo Brasil e por países abaixo da linha do equador.
\end{abstract}

Palavras-chave: Políticas científicas. Produção científica brasileira. Relações conjunturais.

\section{BRAZILIAN SCIENTIFIC PRODUCTION ON THE WEB OF SCIENCE (2017-2019): BETWEEN POLITICAL DISCOURSE AND INFORMATION METRIC STUDIES}

\begin{abstract}
It presents the characteristics of the Brazilian scientific production represented in the Web of Science (WOS) as an illustrative example resulting from the relations between structural and conjunctural elements in the dynamics of the scientific policies adopted by the government in relation to Brazilian science. Methodologically it supported the relational study of the scientific field in Brazil, opting for the use of conjunctural analysis in order to understand the diversity of empirical elements representative of the Brazilian scientific production indexed in the WOS, between 2017 and 2019. It analyses how the productivity demands of development agencies have been intensified in recent decades and are present in discussions in different fields of knowledge, arguing that the rigid practices employed in the field of science justify studies on the correlations and interactions into the favour of understanding and respect for the field and institutions in the fields which is founded. It presents as results, from the empirical data, the formation of five large clusters among which stands out the University of São Paulo (USP) as a central node and centre of national scientific production. Recognizes that, although the chosen database represents the internationalized big science and centralized in the production from the United States and European countries, brazilian representativeness is relevant, especially considering the topics of endemic interest such as tropical diseases and which are researched predominantly by Brazil and by countries below the Equator.
\end{abstract}

Keywords: Keywords: Scientific policies. Brazilian scientific production. Conjunctural relations.

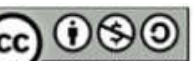


[...] parece inteiramente desejável reforçar a capacidade coletiva de resistência que os pesquisadores devem ter, apesar das concorrências e dos conflitos que os opõem, para estar em condições de resistir às intervenções mais ou menos tirânicas dos administradores científicos e de seus aliados no mundo dos pesquisadores $[\ldots]$ PIERRE BOURDIEU, 2004, p.61, grifo nosso.

\section{INTRODUÇÃO}

Como é de conhecimento público, as melhores universidades do Brasil são públicas e gratuitas e, segundo avaliação divulgada oficialmente pelo próprio Ministério da Educação (MEC), os índices revelam também que entre as 50 melhores, 46 são instituições públicas. Segundo as Notas Estatísticas do Censo de Educação Superior de 2016, 85\% dos docentes na rede pública situam-se no regime de tempo integral, enquanto a rede privada conta apenas com $25,7 \%$ nesse regime de trabalho. Na rede pública, 39\% do corpo docente têm formação de doutorado, índice que cai para 22,5\% na rede privada (BRASIL, 2017).

As contribuições concretas da universidade pública para o desenvolvimento da educação superior, da pós-graduação, da ciência e da inovação tecnológica têm contribuído para que o Brasil ganhe destaque no cenário mundial, segundo evidenciam os dados de pesquisas internacionais. Somente universidades públicas brasileiras estão entre as 20 melhores instituições de ensino e pesquisa da América Latina (FERNANDES, 2018). São elas que investem de modo efetivo na formação de docentes atuantes no país e no exterior, assim como na infraestrutura de laboratórios e hospitais, contribuindo para a universalização e a democratização do acesso ao ensino.

A mobilidade das estruturas universitárias públicas, tanto em prol da interiorização de suas atividades, quanto de seu relacionamento interinstitucional e internacional, amplia sua rede de influência e de investimento. Elas chegam, dessa forma, a alcançar níveis de excelência, não só na qualidade, mas, de modo especial, na extensão de seu desempenho, pois, entre seus estudantes, $51,4 \%$ são oriundos de famílias com renda bruta de até três salários mínimos, segundo pesquisa do Fórum Nacional de Pró-Reitores de Assuntos Comunitários e Estudantis (FONAPRACE, 2016), que também destaca apenas 10,6\% dos estudantes como integrantes de famílias com renda bruta superior a dez salários mínimos.

Além disso, ao promoverem a ampliação de significados no convívio acadêmico, diversamente de suas congêneres no setor privado, as universidades públicas respondem ao princípio da indissociabilidade ensino-pesquisa e extensão, preceito constitucional para as 
universidades brasileiras, assim como ao desenvolvimento de ações sistemáticas em conjunto com a comunidade. As virtudes desse modelo de ensino público destacam-se em especial no contexto posterior à Constituição Federal de 1988, quando se estabelece formalmente o princípio como exigência para as universidades. Ensejando o aprofundamento das discussões sobre o ensino superior e as universidades públicas diante dos apelos democráticos, essa abertura amplia o diálogo com distintos setores da sociedade, em prol da produção de conhecimentos socialmente relevantes e da formação acadêmica articulada com demandas sociais de pesquisa. Essa conjuntura decorre de um esforço histórico para a construção do conhecimento, proveniente, não apenas de iniciativas favoráveis e trabalho efetivo, como também de lutas políticas em prol de sua consolidação e permanência pois todas essas ações requerem investimentos constantes em hospitais, clínicas, museus, teatros e laboratórios, além de equipamentos e material de consumo. Diante do contexto apresentado a pergunta de partida, que orientou a realização da presente pesquisa, foi assim estruturada: as Instituições de Ensino Superior (IES) brasileiras não apresentam contribuições científicas compatíveis com o gasto governamental ao ponto de serem taxadas como locais de muita balbúrdia e pouca ciência? Com o intuito de responder a esse questionamento, buscou-se no objetivo geral verificar as características da produção científica brasileira representada na Web of Science (WOS)?

\section{AS INSTITUIÇÕES DE ENSINO SUPERIOR}

As Instituições de Ensino Superior (IES) apresentam não só os dois polos discursivos (interlocutores e antagonistas) de qualquer pesquisador iniciante, mas também proporcionam investimentos de tempo e de recursos para o desenvolvimento pleno de seu capital científico temporal individual. A construção dessa díade, formada pela união entre a IES e o pesquisador, embasa as relações de poder tecidas entre os agentes e garante a sustentação de ambos, pois é sabido que a dedicação de qualquer membro é proporcional à dependência vital da instituição à qual ele está vinculado, em outras palavras, quem faz a IES é o pesquisador, mas sem ela sua própria existência estaria comprometida. Para a Sociologia da Ciência são as instituições que garantem a organização e as estruturas que viabilizam as trocas científicas. Como bem afirmam Shinn e Ragouet (2008, p. 18)

Graças à atribuição de prêmios e à instauração de um sistema de retribuição, as academias contribuíram para o desenvolvimento de normas, para a ancoragem social dos modelos de excelência e para a instauração de uma hierarquia no interior das comunidades científicas. 
Segundo Schwartzman (1987), ao refletir sobre as diferenças de conteúdo e as "subculturas" científicas, com seus procedimentos de verificação e demonstração, seus padrões de trabalho, suas formas de comunicação, e a maneira de se relacionarem com outras disciplinas e instituições de trabalho científico, a maior contribuição de Joseph Ben-David para os estudos sociológicos da Ciência são

[...] seus estudos históricos sobre as universidades e o contexto cultural, político e institucional do surgimento e das transformações da ciência moderna. Para ele, a ciência moderna requer, para seu desenvolvimento e continuidade, a constituição de um "papel social" para os cientistas, através do qual sua atividade seja reconhecida, prestigiada e protegida. (SCHWARTZMAN, 1987, p. 68 grifo nosso).

A compreensão desse "papel social", pelo qual os pesquisadores têm sua atividade valorizada e mesmo "protegida", envolve o estudo e reconhecimento de sua própria atuação. Em artigo recente publicado no jornal Zero Hora, Itamar Melo (2019) expõe, na amplitude do termo, o que nos difere de países desenvolvidos e com alta representatividade no campo científico. A partir de dados do Censo da Educação Superior de 2017, o autor informa que somente $24,7 \%$ dos universitários brasileiros estavam matriculados em uma instituição governamental (somando federais, estaduais e municipais). A proporção é bem mais expressiva em países como Eslováquia (95\%), Austrália (94\%), Polônia (93\%), Hungria (90\%), Suécia (87\%), Noruega (84\%) e Estados Unidos (67\%). Em que pese o fato de que o Brasil teve seu desenvolvimento científico tardio, comparativamente a esses outros países, o investimento no ensino superior é menos da metade do americano (47,53\%). Outro fator importante quando se fala do Censo da Educação Superior (2017) é que ele aponta para uma prática peculiar ao Brasil que é a incorporação dos servidores inativos na conta dos investimentos realizados. Aproximadamente, $1 / 3$ do custo efetivo por aluno é utilizado para pagamento de aposentadorias que, em princípio, deveriam ser alocadas na conta previdenciária e jamais na de investimento em Educação.

\section{A PÓS-GRADUAÇÃo E O SISTEMA DE PRODUÇÃO CIENTÍFICA}

No que concerne ao modus operandi da pós-graduação no país, ele se constitui como processo formativo e de iniciação do aluno no campo acadêmico e se dá - primeiramente - por meio da articulação e concessão do capital social e científico de seu orientador (BOURDIEU, 2004). Essa relação, em especial, busca não só a inclusão de um novo membro no campo 
(mediante a aprovação dos pares, seja na avaliação de trabalhos ao longo da titulação, seja na outorga do diploma), mas também o prestígio científico aferido aos docentes e aos cursos stricto sensu.

Os pesquisadores brasileiros que na maior parte dos casos também são professores de ensino superior, acabaram por conformar-se ao sistema "publish or perish" (WILSON, 1942 apud GARFIELD, 1996, p.11), ensejado no pós-guerra pelos Estados Unidos como forma de garantir maiores conhecimentos em determinados campos do saber - em especial aos de importância militar, incorporando-o em seu fazer científico de maneira naturalizada. É inegável que, principalmente para as agências de fomento brasileiras, os critérios quantitativos de publicação ainda - mesmo depois de mais de sete décadas - são utilizados como sinônimos de qualidade, seja no nível individual, seja no âmbito institucional.

Essa concepção, aceita e ratificada pela práxis da produção acadêmica, gera - ao mesmo tempo - certo desconforto, ou no mínimo, inquietações à comunidade acadêmica, entre elas a de como conciliar excelência na execução dos três pilares (ensino, pesquisa e extensão) que sustentam a universidade brasileira. É importante salientar, que nos últimos anos (2003-2016), houve expressivo aumento na oferta de vagas e o número de alunos matriculados quase triplicou, de pouco mais de 500 mil para mais de 1,3 milhão. "Isso abriu as portas da universidade a milhões de brasileiros e, consequentemente, significou gastos mais pesados para o Ministério da Educação (MEC)" (MELO, 2019).

É fato que as exigências produtivistas das agências de fomento intensificaram-se nas últimas décadas e estão presentes em inúmeras discussões nos mais diferentes campos do saber. Em 1988, um artigo intitulado "Pesquisa da USP mostra que 1/4 dos docentes nada produz", publicado na Folha de São Paulo, causou desconforto (indignação talvez seja a palavra mais adequada) entre os professores universitários, em que pese o fato de as declarações não terem impactado somente nesse universo, mas sim, comovido a classe docente em âmbito nacional.

Cabe contextualizar essa discussão, pois foi emblemática para o que aqui se discute. Ao mencionar que 25\% dos docentes nada produziram, entre 1985-1986, a Folha de São Paulo (e a Reitoria) os rotulou como "os improdutivos da USP” (FOLHA, 1988, p. 2, grifo nosso) esse enunciado surgia, no Brasil, pela primeira vez em um veículo de comunicação de significativa expressão. Ao descreverem, em tom de denúncia, a "improdutividade" dos professores, os articulistas afirmaram que é papel da universidade difundir o conhecimento, ou seja, publicá-lo seria imprescindível para o devido cumprimento dessa função. Em sendo assim, como esse percentual representativo de professores não o fez em dois anos? 
Infelizmente, nem os jornalistas, nem a Reitoria utilizaram métricas adequadas para mensurar (condenar) 25\% dos professores. Nessa conta não foram consideradas as horas em sala de aula, os cargos de gestão e representação ocupados, nem ao menos o tempo médio (por área) entre a pesquisa e a publicação dela. É por esse motivo que muitas das críticas aos métodos quantitativos de análise sejam alardeadas, como fica patente no diálogo reproduzido de uma reunião da Associação dos Docentes da Universidade Federal de São Paulo (ADUSP): “A cientometria pode ter consequências desastrosas. Medidas em geral afetam a grandeza que a gente está medindo [...] a citação virou moeda de troca: eu lhe cito, você me cita. Escambo autoral” (BURIAN JR., 2011, p. $3^{1}$ ).

Ora, os estudos métricos não estão ou pelo menos não deveriam estar restritos à descrição do cenário (medidas generalistas) encontrado no campo, mas sim, alinhavando indicadores quantitativos com teorias que se proponham a contextualizar e historicizar os resultados encontrados. Em sendo assim, pergunta-se como romper com algo que é ao mesmo tempo criticado por grande parte dos membros do campo e, em contrapartida, ratificado por um pequeno grupo de porta-vozes autorizados?

Solla Price (1976) afirmou que fazer ciência sobre a Ciência é um dos caminhos para um melhor entendimento acerca da sua institucionalização. Considerando-se a atividade científica uma realidade passível de ser representada parcialmente em termos de quantidades, para que possa ser analisada em suas tendências mais globais e produzir inferências significativas tanto sobre sua natureza expansiva, quanto sobre os possíveis efeitos redutores de seu crescimento. os estudos métricos têm como intento primeiro apontar como determinadas variáveis influenciam a produção científica de determinado país ou de qualquer outro recorte geográfico. As análises resultantes da aplicação dessa metodologia auxiliam a alocação de recursos, permitem identificar a necessidade de correção/alteração de rumo no campo científico, mas, sobretudo, indicam quem são os principais agentes constituintes, quais são as temáticas mais relevantes e/ou incipientes, etc.

\footnotetext{
${ }^{1}$ Informação oral. BURIAN JÚNIOR, Yaro. Choque de visões antagônicas marca debate sobre produtivismo acadêmico. Informação disponível em: http://www.adusp.org.br/index.php/universidade2/180-condicoes-detrabalho/produtivismo/953-choque-de-visoes-antagonicas-marca-debate-sobre-produtivismo-academico. Acesso em: 02 set. 2019.
} 


\section{PERCURSO METODOLÓGICO}

O estudo analisa a produção científica brasileira indexada na WOS, entre os anos de 2015 e 2018. Ao propor uma análise relacional do campo científico no Brasil, enseja uma melhor compreensão do contexto da pesquisa no país. Para tanto, optou-se pela utilização da análise de conjuntura para não só facilitar a compreensão dos dados quantitativos, mas também - e principalmente - contextualizar as informações qualitativas inscritas no contexto do fenômeno.

É sabido que, com o volume de informações veiculadas diariamente, é “[...] necessário identificar os ingredientes, os atores [agentes], os interesses em jogo [enjeux]" (SOUZA, 2004, p. 7). Em função da complexidade do problema proposto, optou-se por fazer esse tipo de análise, pois ela promove a interação entre a descoberta e o conhecimento prévio sobre determinado fato.

O cume da arte, em ciências sociais, está sem dúvida em ser-se capaz de por em jogo coisas teóricas muito importantes a respeito de objetos ditos empíricos [...] Tem-se demasiada tendência para crer [...] que a importância social ou política do objeto é por si mesmo suficiente para dar fundamento à importância do discurso que lhe é consagrado - é isto sem dúvida que explica que os sociólogos [...] se mostrem muitas vezes os menos atentos aos procedimentos metodológicos (BOURDIEU, 2011, p. 18).

Em relação aos acontecimentos geradores do estudo, ressalta-se a efervescente discussão sobre os investimentos em Educação Superior no Brasil; o cenário se constituiu a partir da trama social e política; já nas relações de forças se pode citar a necessidade de coexistência, ou melhor, resistência das IES frente aos cortes orçamentários de toda ordem (custeio, bolsas e etc.) e, por fim, intenta-se apresentar a articulação entre a estrutura e a conjuntura atual.

Técnicas cientométricas foram aplicadas pelas visualizações mais expressivas dos dados obtidos na WOS. Embora reconhecendo as inúmeras críticas à aplicação de estudos métricos para a explicação de fenômenos sociais, assume-se a sua adoção, considerando os cuidados para evitar o que aponta Bourdieu (2004) como o emprego das técnicas métricas, em desalinho a uma análise social, o que funcionaria como ratificador das posições de poder, uma vez que os critérios de avaliação são propostos pela cúpula dominante. Por esse motivo justifica-se a escolha do emprego da análise de conjuntura e de técnicas cientométricas para um melhor entendimento do problema que norteou o presente estudo.

A pesquisa na WOS se deu no dia 02 de setembro de 2019 com a seguinte estratégia de busca: ENDEREÇO: (brasil) OR ENDEREÇO: (brazil) Tempo estipulado: 2017-2019. Índices: 
SCI-EXPANDED, SSCI, A\&HCI, CPCI-S, CPCI-SSH, ESCI. O universo revocado foi de 204570 trabalhos (artigos, anais, capítulos de livro, material editorial entre outros). Optou-se pela aplicação dos seguintes filtros: artigo (162205) e exclusão de instituições estrangeiras (142840).

Para gerar os grafos utilizou-se o software VOSViewer 1.6.11 da Universidade de Leiden, na versão 1.6.11. O layout escolhido foi o de linlog/modularity e foram mantidas as linhas que tinham força de ligação (strenght) cinco ou superior, além de definir a variação de tamanho (size variation) das linhas no máximo (1.00) essas escolhas tiveram como objetivo conferir maior clareza aos vínculos apresentados.

\section{RESULTADOS E ANÁLISES}

Dos 142840 artigos que compuseram o universo da pesquisa $64,84 \%$ deles foram financiados por sete das principais agencias públicas de fomento como pode ser observado na tabela a seguir.

Tabela 1 - Pesquisas financiadas por agências públicas de fomento (2017-2019)

\begin{tabular}{lrr}
\hline Agência & $\begin{array}{r}\text { Frequência absoluta de artigos } \\
\text { indexados na WOS }\end{array}$ & \% de artigos indexados na WOS \\
\hline CNPq & 41223 & $28,86 \%$ \\
CAPES & 27008 & $18,90 \%$ \\
FAPESP & 16296 & $11,41 \%$ \\
FAPEMIG & 4361 & $3,05 \%$ \\
FAPERJ & 1897 & $1,33 \%$ \\
FINEP & 982 & $0,69 \%$ \\
FAPERGS & 862 & $0,60 \%$ \\
OUTRAS & 51622 & $35,16 \%$ \\
TOTAL & $\mathbf{1 4 2 8 4 0}$ & $\mathbf{1 0 0 , 0 0 \%}$ \\
\hline
\end{tabular}

Fonte: dados da pesquisa (2019).

É importante salientar que esse percentual expressivo de artigos publicados por meio de financiamento público é fruto da destinação de verbas de fomento às principais agências do país. A implementação de uma política científica, que engloba não só a pesquisa, mas o ensino de graduação e de pós-graduação, encontra nesses dados a maior justificativa para o tamanho do investimento governamental. Não há ciência no país se não houver investimento público e fortalecimento das IES que têm, em seus laboratórios e núcleos, a maior parte da produção científica nacional.

O Brasil ao longo dos últimos anos, tem se apresentado como o $13^{\circ}$ maior produtor de publicações no mundo e tem aumentado, nos últimos 6 anos, em 15\% seu impacto na Ciência 
(CLARIVATES ANALYTICS, 2018). Ainda em relação à performance brasileira o relatório aponta as três grandes Universidades em termos de volume de produção, a saber: USP, UFRJ e UNICAMP.

Tabela 2 - Distribuição da produção científica brasileira entre as áreas do conhecimento (2017-2019)

\begin{tabular}{lrr}
\hline Áreas de pesquisa & Freq. Abs. & Freq. Rel. (\%) \\
\hline ENGINEERING & 11260 & $7,88 \%$ \\
AGRICULTURE & 11095 & $7,67 \%$ \\
CHEMESTRY & 10508 & $7,35 \%$ \\
ENVIRONMENTAL SCIENCES ECOLOGY & 7331 & $5,13 \%$ \\
MATERIALS SCIENCE & 6468 & $4,52 \%$ \\
SCIENCE TECHNOLOGY OTHER TOPICS & 6250 & $4,37 \%$ \\
PHYSICS & 6005 & $4,20 \%$ \\
BIOCHEMISTRY MOLECULAR BIOLOGY & 4453 & $3,11 \%$ \\
PLANT SCIENCES & 4287 & $3,00 \%$ \\
MATHEMATICS & 4235 & $2,96 \%$ \\
FOOD SCIENCE TECHNOLOGY & 4070 & $2,84 \%$ \\
PUBLIC ENVIRONMENTAL OCCUPATIONAL HEALTH & 3957 & $2,77 \%$ \\
COMPUTER SCIENCE & 3921 & $2,74 \%$ \\
BUSINESS ECONOMICS & 3844 & $2,69 \%$ \\
EDUCATION EDUCATIONAL RESEARCH & 3781 & $2,65 \%$ \\
PHARMACOLOGY PHARMACY & 3776 & $2,64 \%$ \\
VETERINARY SCIENCES & 3624 & $2,53 \%$ \\
DENTISTRY ORAL SURGERY MEDICINE & 3506 & $2,45 \%$ \\
ZOOLOGY & 3466 & $2,42 \%$ \\
NEUROSCIENCES NEUROLOGY & 3095 & $2,16 \%$ \\
MICROBIOLOGY & 2270 & $1,58 \%$ \\
BIOTECHNOLOGY APPLIED MICROBIOLOGY & 2214 & $1,55 \%$ \\
NURSING & 2158 & $1,51 \%$ \\
NUTRITION DIETETICS & 2122 & $1,48 \%$ \\
MARINE FRESHWATER BIOLOGY & 2075 & $1,43 \%$ \\
OTHERS & 23069 & $16,15 \%$ \\
TOTAL & 142840 \\
\hline For & \\
\hline
\end{tabular}

Fonte: dados da pesquisa (2019)

Em relação às Instituições de Ensino e Pesquisa, mais profícuas no período o que se pode observar, é a predominância de IES públicas (91,54\%) dos 142840 artigos indexados na WOS no período de 2017-2019. 
Tabela 3 - Ranking das Instituições brasileiras mais profícuas representadas na WOS (2017-2019)

\begin{tabular}{llrr}
\hline & Organizações & Freq. Abs. & Freq. Rel. (\%) \\
\hline $1^{\mathrm{a}}$ & USP & 24127 & $16,89 \%$ \\
$2^{\mathrm{a}}$ & UNESP & 10606 & $7,43 \%$ \\
$3^{\mathrm{a}}$ & UNICAMP & 8546 & $5,98 \%$ \\
$4^{\mathrm{a}}$ & UFMG & 7222 & $5,06 \%$ \\
$5^{\mathrm{a}}$ & UFRJ & 7145 & $5,00 \%$ \\
$6^{\mathrm{a}}$ & UFRGS & 7039 & $4,93 \%$ \\
$7^{\mathrm{a}}$ & UFPR & 5715 & $4,00 \%$ \\
$8^{\mathrm{a}}$ & UFSC & 4858 & $3,40 \%$ \\
$9^{\mathrm{a}}$ & UNIFESP & 4524 & $3,17 \%$ \\
$10^{\mathrm{a}}$ & EMBRAPA & 4234 & $2,97 \%$ \\
$11^{\mathrm{a}}$ & UFSCAR & 3720 & $2,60 \%$ \\
$12^{\mathrm{a}}$ & UFSM & 3706 & $2,59 \%$ \\
$13^{\mathrm{a}}$ & FIOCRUZ & 3674 & $2,57 \%$ \\
$14^{\mathrm{a}}$ & UnB & 3596 & $2,52 \%$ \\
$15^{\mathrm{a}}$ & UFPE & 3593 & $2,51 \%$ \\
$16^{\mathrm{a}}$ & UFC & 3293 & $2,30 \%$ \\
$17^{\mathrm{a}}$ & UFV & 3266 & $2,29 \%$ \\
$18^{\mathrm{a}}$ & UNIRIO & 3035 & $2,13 \%$ \\
$19^{\mathrm{a}}$ & UFF & 3002 & $\mathbf{1 0 0 , 0 0 \%}$ \\
$20^{\mathrm{a}}$ & UFG & $2,10 \%$ \\
$21^{\mathrm{a}}$ & UFMS & 12069 & \\
$22^{\mathrm{a}}$ & UFRN & $2,07 \%$ \\
$23^{\mathrm{a}}$ & UFBA & 2959 & $2,03 \%$ \\
$24^{\mathrm{a}}$ & UFPB & 2908 & $1,93 \%$ \\
$25^{\mathrm{a}}$ & UEM & 2757 & $1,75 \%$ \\
& OUTRAS & $1,70 \%$ \\
& TOTAL & 2495 & $1,62 \%$ \\
\hline
\end{tabular}

Fonte: dados da pesquisa (2019)

Considerando o exposto no relatório solicitado à Clarivates Analytics pela CAPES, a produção científica brasileira desenvolveu-se acima do esperado, principalmente, tendo em vista que o ano de 2019 ainda está em curso (CLARIVATES ANALYTICS, 2018). Ainda de acordo com o documento, a perspectiva é de que se o Brasil mantivesse seu crescimento, não só em volume, mas principalmente, em impacto (número de citações recebidas por artigo) alcançaríamos a mesma média mundial que é de 1,0 em 2021. "Between 2011 and 2016 Brazil's citation impact remained below the world average. However, Brazil's impact has increased year-on-year from 0.73 in 2011 to 0.86 in 2016, a rise of $18 \%$. If this current trend is maintained 
then by 2021 Brazil will have reached the global average of 1.0" (CLARIVATES ANALYTICS, 2018, p. 9).

Outro ponto importante da tabela anterior, é a hegemonia das instituições que compõem o eixo sul-sudeste entre as 10 primeiras colocadas. O Plano Nacional da Pós-Graduação (PNPG 2011-2020) visando diminuir a assimetria regional verificada definiu, por meio de implantação de política pública de fundos setoriais, a destinação de 30\% dos recursos para as regiões Norte, Nordeste e Centro-Oeste (CAPES, 2010).

Por meio do software VOSViewer, foi possível visualizar as colaborações interinstitucionais, a partir da amostra dos 15000 artigos mais citados entre os 142840 revocados.

Grafo 1 - Coautorias entre as Instituições de pesquisa brasileiras (2017-2019)

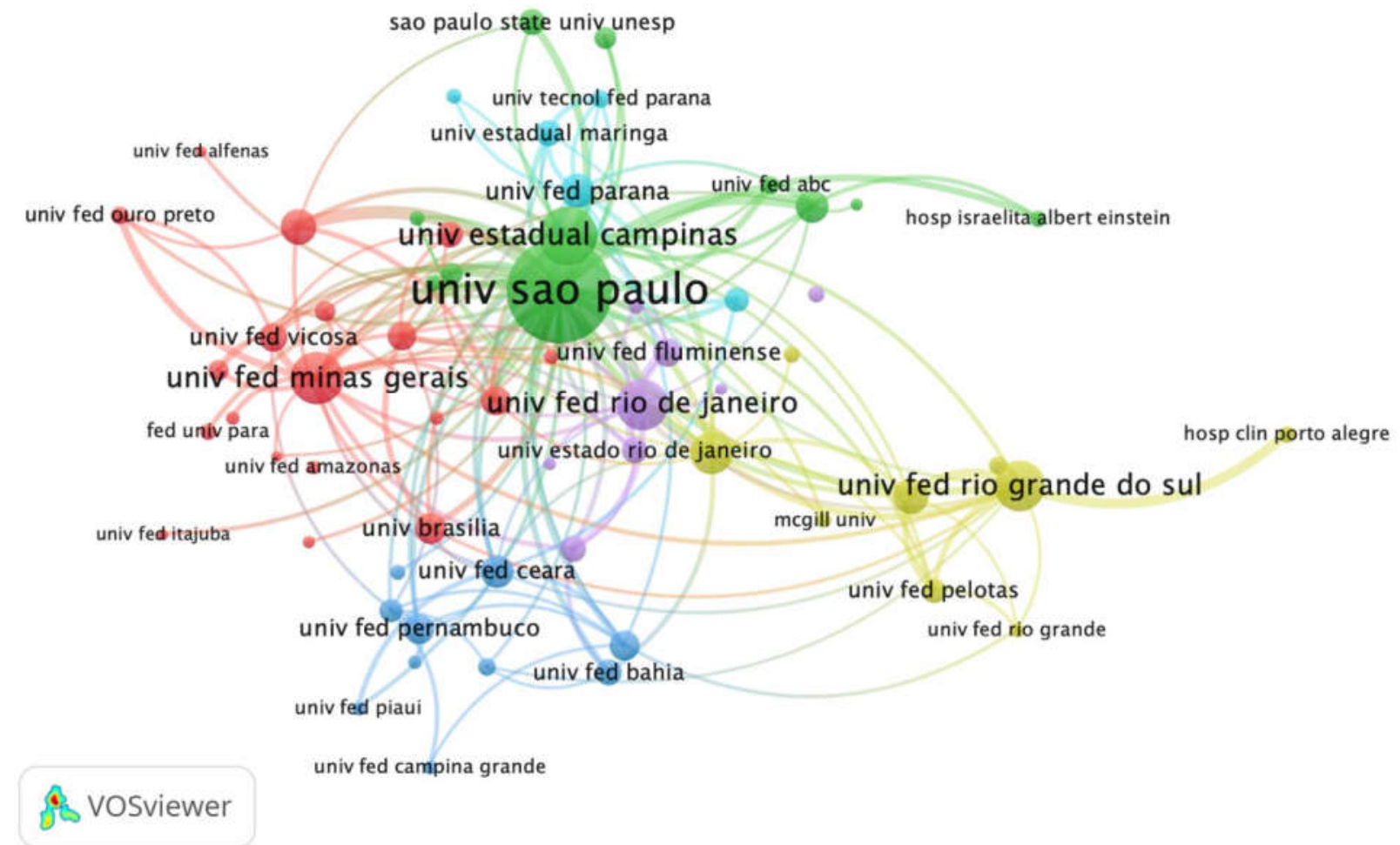

Fonte: dados da pesquisa (2019)

O que se pode notar ao analisar o grafo é a formação de cinco grandes clusters com a relevância demonstrada pela diferenciação cromática, a saber: verde, vermelho, amarelo, lilás e azul. O cluster verde com maior número de artigos publicados, estrutura-se tendo a Universidade de São Paulo (USP) como nó central da microrrede. É importante salientar que 
31 anos depois do famigerado artigo da Folha de São Paulo a Universidade desponta como o grande polo de produção científica nacional.

Em que pesem as limitações da base de dados escolhida, pois ela é proprietária e, por conseguinte, sua representação está atrelada à big science internacionalizada e centralizada na produção oriunda dos Estados Unidos e de países europeus, a representatividade brasileira não pode ser considerada baixa ou irrelevante, tendo em vista que muito de sua produção em ciência sustenta-se em temáticas de interesse endêmico como, por exemplo, as doenças tropicais e que são pesquisadas, predominantemente, pelo Brasil e por países abaixo da linha do equador.

\section{CONSIDERAÇÕES FINAIS}

Destacam-se, como considerações finais, aspectos significativos especialmente decorrentes das relações entre instituições e acontecimentos, no cenário brasileiro, em prol da articulação possível entre estrutura e conjuntura. Essas relações sugerem, em suas expressões observáveis no cotidiano, as condições determinantes das produções científicas e suas repercussões, decorrentes dos processos de comunicação, da sua estrutura administrativa e dos instrumentos de gestão e avaliação consolidados nas práticas de seus agentes. Nessa conjuntura, são discutidas, construídas e consolidadas concepções de universidade, de seus papeis de Ensino, de Pesquisa e de Extensão.

No que concerne à metodologia empregada, acredita-se as planilhas, geradas a partir do Excel, auxiliaram na padronização dos dados. Quanto à escolha do software VOSViewer, para a confecção do grafo de colaboração interinstitucional que demonstrou as posições de centralidade e poder entre essas instituições. As escolhas demonstraram estar de acordo com as expectativas e atenderam às necessidades da investigação.

Uma possibilidade, para a expansão das análises aqui apresentadas, diz respeito ao cotejamento dos dados de produção científica (WOS), mas com a ampliação do corpus incluindo outra grande base de dados como, por exemplo, a SCOPUS. Isso permitirá um melhor entendimento de como a ciência brasileira se constitui e se internacionaliza.

Os estudos, como o aqui esboçado, servem para elucidar as relações entre elementos estruturais e conjunturais que (in)viabilizam, a depender das políticas científicas adotadas pelo governo, a ciência brasileira. Mesmo diante das rígidas práticas empregadas no campo da Ciência, identificar as correlações e interações auxiliam na tomada de consciência a respeito de como o campo se edifica e em quais instituições ele se alicerça. 
Portanto, a título de conclusão, resta o apelo a pessoas, movimentos e instituições para que se unam em prol das instituições públicas de pesquisa e pela democratização do saber, fazendo ouvir sua voz, na luta contra a entrega do país a interesses obscuros que operam à margem da lei e da democracia. 


\section{REFERENCIAS}

BOURDIEU, Pierre. O poder simbólico. Lisboa: Edições 70, 2011.

BOURDIEU, Pierre. Os usos sociais da ciência: por uma sociologia do campo científico. São Paulo: UNESP, 2004.

BRASIL, Instituto Nacional de Estudos e Pesquisas Educacionais Anísio Teixeira - INEP. Censo da Educação Superior no Brasil 2016: Notas Estatísticas. Ministério da Educação, Brasília, 2017. Disponível em:

http://download.inep.gov.br/educacao_superior/censo_superior/documentos/2016/notas_sobre _o_censo_da_educacao_superior_2016. Acesso em 24/07/2019.

BRASIL. Ministério da Educação, 2016. Censo da educação superior. Brasília: INEP. Disponível em:

http://download.inep.gov.br/educacao_superior/censo_superior/documentos/2016/notas_sobre _o_censo_da_educacao_superior_2016.jpdf. Acesso em: 27 jul. 2019.

CLARIVATES ANALYTICS. Research in Brazil: a report for CAPES by Clarivate Analytics, 2018. Disponível em:

https://www.capes.gov.br/images/stories/download/diversos/17012018-CAPES-

InCitesReport-Final.pdf. Acesso em: 24 ago. 2019.

FERNANDES, Marcella. Universidades brasileiras lideram ranking da Times Higher Education na América Latina. Disponível em:

https://www.huffpostbrasil.com/2018/07/18/brasil-lidera-ranking-das-universidades-daamerica-latina-da-times-higher-education_a_23483884/. Acesso em: 27 jul. 2019.

FOLHA DE SÃO PAULO. Pesquisa da USP mostra que 1/4 dos docentes nada produz. Primeiro Caderno, São Paulo, 1988. Disponível em:

$<$ http://acervo.folha.uol.com.br/resultados/buscade_talhada/?utf $8=0 \mathrm{E} 2 \% 9 \mathrm{C} \% 93 \& \mathrm{fsp}=$ on\&all _words $=\&$ phrase $=\&$ words $=$ usp \&without_words $=\&$ initial_date $=21 \% 2 \mathrm{~F} 02 \% 2 \mathrm{~F} 1988 \&$ final_da 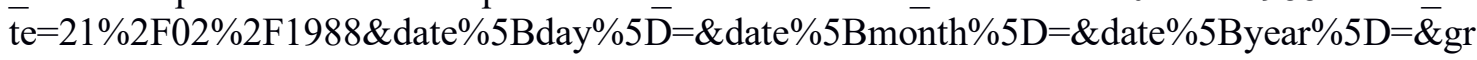 oup_id $=0 \&$ theme_id $=0 \&$ commit. $x=38 \&$ commit. $y=16 \&$ commit $=$ Enviar $>$. Acesso em: 24 jul. 2019 .

FONAPRACE, Revista Comemorativa 25 Anos histórias, memórias e múltiplos olhares. FONAPRACE - Fórum Nacional de Pró-reitores de Assuntos Comunitários e Estudantis ANDIFES - Associação Nacional dos Dirigentes das Instituições Federais de Ensino Superior, 2016.

GARFIELD, Eugene. "What Is The Primordial Reference For The Phrase 'Publish Or Perish'?", The Scientist, v. 10, n. 12, jun. 1996. 
GAUCHAZH. Educação e Trabalho. Disponível em:

https://gauchazh.clicrbs.com.br/educacao-e-emprego/noticia/2019/05/o-brasil-gasta-demaiscom-universidade-publica-veja-numeros-e-comparacao-com-outros-paisescjw6ja81x00ad01s9z9iyrizh.html Acesso em: 18 ago. 2019.

SCHWARTZMAN, S. Relatório de uma visita ao brasil. Ciência Hoje, São Paulo, v. 7, n. 37, nov., 1987. Disponível em: <http://www.schwartzman.org.br/simon/bendavid.htm>. Acesso em: 12 out. 2015.

SHINN, Terry; RAGOUET, Pascal. Controvérsias sobre a ciência: por uma sociologia transversalista da atividade científica. São Paulo: Ed. 34, 2008.

SOLLA PRICE, Derek J. de. O Desenvolvimento da Ciência: análise histórica, filosófica, sociológica e econômica. Rio de Janeiro: Livros Técnicos e Científicos, 1976. Tradução de Little science big science.

SOUZA, Betinho. Análise de conjuntura. 25. ed. Petrópolis: Vozes, 2004. 